\title{
Correlation between high perfusion syndrome and stent restenosis after stent implantation
}

\author{
YINGYI LI ${ }^{1}$, LINGTAO TANG ${ }^{1}$, DONG $^{1}{ }^{1}$, CHUNLEI WANG $^{2}$, SUXIA ZHANG $^{1}$, \\ PENGFEI HU ${ }^{1}$, YUN WANG ${ }^{1}$, BOGANG ZHANG ${ }^{1}$ and KUNXI ZHANG ${ }^{1}$ \\ ${ }^{1}$ Department of Neurology, The Third Hospital of Xingtai City; ${ }^{2}$ Department of Neurology, \\ People's Hospital of Wei County, Xingtai, Hebei 054000, P.R. China
}

Received July 13, 2016; Accepted September 19, 2016

DOI: 10.3892/etm.2016.3813

\begin{abstract}
The present study was conducted to determine the correlation between high perfusion syndrome and stent restenosis after cerebral vascular stent implantation. A total of 146 patients diagnosed with cerebral vascular stenosis and stent implantation were selected. A total of 55 cases (37.67\%) of cerebral hyperperfusion syndrome patients were diagnosed by xenon-enhanced computer tomography (Xe-CT) examination and clinical symptoms within 3 days after surgery and were chosen as the observation group. A total of 91 cases were selected as the control group. After treatment, blood flow of the anterior cerebral artery, middle cerebral artery, posterior cerebral artery, anterior border zone, posterior border zone and the inner border zone of the two groups increased, with values in the observation group increasing more significantly, and the differences were statistically significant $(\mathrm{P}<0.05)$. The rate of restenosis and target lesion diameter one month and one year after operation in the observation group were significantly higher than those in the control group $(\mathrm{P}<0.05)$. Multivariate logistic regression analysis showed that the mean systolic blood pressure (mSBP), mean diastolic blood pressure (mDBP), stenosis rate of cerebral vascular diameter and high perfusion syndrome were independent risk factors for restenosis $(\mathrm{P}<0.05)$. The application of Xe-CT examination is important for early diagnosis of hyperperfusion syndrome. Hyperperfusion syndrome and the occurrence of stent restenosis are closely related. $\mathrm{mSBP}, \mathrm{mDBP}$, cerebral blood vessel diameter stenosis rate and high perfusion comprehensive syndrome are the independent risk factors of restenosis.
\end{abstract}

Correspondence to: Dr Kunxi Zhang, Department of Neurology, The Third Hospital of Xingtai City, 108 North Steel Road, Xingtai, Hebei 054000, P.R. China

E-mail: sp7563818yishibi@163.com

Key words: cerebral vascular stent implantation, high perfusion syndrome, stent restenosis

\section{Introduction}

Cerebrovascular disease is a common disease with a high incidence rate, a high disability rate, high recurrence rate and high mortality (1). Approximately $70 \%$ of the patients lose working and self-care ability, and it is a serious threat to the health and quality of life of the elderly population. Cerebral vascular stent implantation can effectively relieve cerebral artery stenosis, but high perfusion syndrome after implantation is a serious complication of carotid artery stenting (2). This occurs mainly due to the rapid increase in perfusion pressure of the microvascular bed which leads to blood-brain barrier damage accompanied by related clinical symptoms, such as intracranial hemorrhage, brain edema, the side of moderate to severe headache, hemiplegia, aphasia and epilepsy (3).

Stent restenosis is also a serious complication (4), which can be diagnosed by computed tomography angiography (CTA). There may be a correlation between cerebral hyperperfusion syndrome (CHS) and ISR, but there is less research on this issue.

The aim of the present study was to analyze the relationship between the two groups, and to analyze the influencing factors in order to provide a reference for clinical early intervention.

\section{Materials and methods}

General information. A total of 146 patients diagnosed with cerebral vascular stenosis and stent implantation were selected in the Third Hospital of Xingtai City from January 2010 to January 2016. All the patients had single target site lesions that led to acute cerebral infarction, with vascular stenosis rate of $>90 \%$, and stent implantation indications, expected to be effective after treatment. Exclusion criteria included patients with cerebral hemorrhage, congenital vascular brain development abnormalities, and the tiny blood vessels without appropriate scaffold types or unable to complete lesion coverage, intraoperative acute stent thrombosis, blood vessel perforation, hemorrhage, brain tumor, recent surgery, blood coagulation dysfunction, serious illness, and an expected survival period of $<12$ months. The study was approved by the Ethics Committee of the Third Hospital of Xingtai City and written informed consent of patients or their families was obtained. Patients were recruited within 3 days after operation 
Table I. Comparison of baseline data between two groups.

\begin{tabular}{lcccc}
\hline Item & Control group, $\mathrm{n}=91$ & Observation group, $\mathrm{n}=55$ & $\mathrm{t} / \chi^{2}$ & P-value \\
\hline Male/female & $52 / 39$ & $33 / 22$ & 0.115 & 0.734 \\
Age, year & $58.8 \pm 7.6$ & $59.6 \pm 7.5$ & 0.230 & 0.685 \\
Time of onset, $\mathrm{h}$ & $8.2 \pm 2.5$ & $8.3 \pm 2.4$ & 0.167 & 0.722 \\
Smoke, cases (\%) & $13(14.29)$ & $8(14.55)$ & 0.002 & 0.965 \\
Diabetes, cases (\%) & $10(10.99)$ & $6(10.91)$ & 0.000 & 0.988 \\
Cholesterol total, mmol/l & $5.7 \pm 0.8$ & $5.8 \pm 0.7$ & 0.245 & 0.623 \\
Low density lipoprotein, mmol/1 & $4.5 \pm 1.0$ & $4.7 \pm 1.2$ & 0.324 & 0.522 \\
BMI, kg/m & $22.4 \pm 2.2$ & $22.6 \pm 2.5$ & 0.335 & 0.503 \\
mSBP, mmHg & $165.7 \pm 4.3$ & $167.2 \pm 4.4$ & 0.267 & 0.642 \\
mDBP, mmHg & $102.4 \pm 5.2$ & $103.6 \pm 5.6$ & 0.423 & 0.527 \\
Cerebral vascular stenosis rate, $\%$ & $96.8 \pm 3.3$ & $97.2 \pm 3.5$ & 0.236 & 0.647 \\
Stent length, mm & $12.3 \pm 3.2$ & $13.4 \pm 3.3$ & 0.326 & 0.632 \\
\hline
\end{tabular}

BMI, body mass index; mSBP, mean systolic blood pressure; mDBP, mean diastolic blood pressure.

by xenon-enhanced $\mathrm{CT}(\mathrm{Xe}-\mathrm{CT})$ examination and clinical symptoms, which confirmed high perfusion comprehensive syndrome in 55 patients $(37.67 \%)$, which were designated the observation group. The remaining 91 cases were designated as the control group, and baseline data of the two groups was comparable (Table I).

Stent implantation. The study was completed by the same surgical and nursing team, based on standard medical procedures. In order to improve preoperative preparation, we used subcutaneous local anesthesia and used Seldinger's puncture technique. We placed the wire into the arterial sheath, guided the wire through to stenosis, along the catheter and put balloon dilatation into the target lesion and slowly increased the pressure in the balloon until it reached a maximum pressure lasting $20 \mathrm{sec}$. After the full expansion, we implanted drug eluting stents and performed stent coverage beyond the narrow section at $5 \mathrm{~mm}$, with routine expansion. After stent placement, the artery sheath was retained for $6 \mathrm{~h}$ and removed. We applied perioperative routine anticoagulation and antiplatelet therapy, and reduced intracranial pressure, controlled blood pressure, blood glucose levels, blood lipids and stabilize internal environment.

Xe-CT check. The PLUS4CT scanner (Siemens AG, Munich, Germany) combined American xenon-CT were used. Patient was held supine with head fixed by fiber glass head fixation. A vacuum bag as a head brake. We first took a CT routine scan of the head, and then xenon mixed gas inhalation with the concentration of $30 \%$ was carried out through the mask. The patients remained awake in order to control the frequency and depth of breathing and to ensure the stability of the respiratory $\mathrm{CO}_{2}$ concentration, in the process of xenon inhalation. Patients were continuously scanned, 6 times, through the basement, we took a Xe-CT scan slice (thickness $10 \mathrm{~mm}$ ), and took the central, lenticular nucleus, caudate nucleus and thalamus level of the lateral ventricle anterior horn of patients as the first layer. We sequentially scanned, getting the images of fourth layer. After the end of the scan, we removed the mask to assess the patients. The observation sites include: i) Anterior cerebral artery (ACA); ii) middle cerebral artery (MCA); iii) Posterior cerebral artery (PCA); iv) anterior border zone (ABZ): Between the ACA and the MCA; v) Posterior border zone (PBZ), located in the MCA and the middle artery blood supply scope, namely the temporal, the top, the occipital border area; and vi) inner border zone (IBZ), located between the cortical branch of MCA and the artery of the bean, the recurrent artery and the anterior choroidal artery, next to the basal ganglia and the lateral ventricles. The cerebral blood flow (CBF) of the observation site abnormally increased and the typical lesions were beaded or linearly changed.

CTA check. We followed up 1 month and 1 year after surgery. We performed a routine CT plain scan, and then the high pressure syringe was injected with $35 \mathrm{ml}$ iodine contrast agent and $0.9 \%$ sodium chloride solution at the speed of $7 \mathrm{ml} / \mathrm{sec}$ and delayed $5 \mathrm{sec}$ for enhanced scan. At the end of the scan, we used multi planar reconstruction, surface reconstruction, maximum density projection, volume display and other techniques for restructuring. We measured the diameter of the narrow blood vessels and normal blood vessels in the stent. Stenosis rate of vascular lumen in stent $\geq 50 \%$ is the standard for restenosis.

Statistical analysis. SPSS 19.0 software (SPSS, Inc., Chicago, IL, USA) was used for data analysis. Quantitative data were expressed as mean \pm standard deviation. The group comparison was tested by independent sample t-test, and comparison within group was tested by paired $\mathrm{t}$-test. Countable data were expressed by the rate and $\chi^{2}$ test was used. A logistic regression model was used to influence factor analysis of stent restenosis. $\mathrm{P}<0.05$ was considered to indicate a statistically significant difference.

\section{Results}

Xe-CT examination result analysis. After treatment, the blood flow of ACA, MCA, PCA, ABZ, PBZ, and IBZ in both groups increased; the observation group increased more significantly, and the differences were statistically significant $(\mathrm{P}<0.05$; Table II). 
Table II. Xe-CT examination results analysis $(\mathrm{ml} /(100 \mathrm{~g} \cdot \mathrm{min})$.

\begin{tabular}{|c|c|c|c|c|}
\hline Group & Control group & Observation group & $\mathrm{t}$ & P-value \\
\hline \multicolumn{5}{|l|}{$\mathrm{ACA}$} \\
\hline Before operation & $35.6 \pm 4.3$ & $34.6 \pm 4.4$ & 0.069 & 0.923 \\
\hline 3 days after operation & $40.5 \pm 5.2$ & $48.9 \pm 5.3$ & 4.632 & 0.037 \\
\hline \multicolumn{5}{|l|}{ MCA } \\
\hline Before operation & $32.4 \pm 3.6$ & $32.3 \pm 3.5$ & 0.072 & 0.914 \\
\hline 3 days after operation & $36.6 \pm 3.7$ & $42.3 \pm 3.9$ & 4.527 & 0.039 \\
\hline \multicolumn{5}{|l|}{ PCA } \\
\hline Before operation & $27.6 \pm 3.2$ & $26.5 \pm 3.3$ & 0.125 & 0.867 \\
\hline 3 days after operation & $32.3 \pm 3.5$ & $37.6 \pm 3.7$ & 5.201 & 0.033 \\
\hline \multicolumn{5}{|l|}{ ABZ } \\
\hline Before operation & $23.3 \pm 3.0$ & $22.4 \pm 3.2$ & 0.135 & 0.822 \\
\hline 3 days after operation & $26.7 \pm 3.2$ & $32.3 \pm 3.3$ & 5.326 & 0.031 \\
\hline \multicolumn{5}{|l|}{ PBZ } \\
\hline Before operation & $21.4 \pm 2.8$ & $20.3 \pm 2.6$ & 0.164 & 0.735 \\
\hline 3 days after operation & $25.4 \pm 2.7$ & $30.5 \pm 2.5$ & 5.526 & 0.026 \\
\hline \multicolumn{5}{|l|}{ IBZ } \\
\hline Before operation & $16.7 \pm 2.3$ & $15.8 \pm 2.4$ & 0.245 & 0.635 \\
\hline 3 days after operation & $18.3 \pm 2.6$ & $25.4 \pm 2.7$ & 5.127 & 0.035 \\
\hline
\end{tabular}

Xe-CT, xenon-enhanced computer tomography; ACA, anterior cerebral artery; MCA, middle cerebral artery; PCA, posterior cerebral artery; $\mathrm{ABZ}$, anterior border zone; PBZ, posterior border zone; IBZ, inner border zone.

Table III. Comparison of the incidence of stent restenosis.

\begin{tabular}{lccccc}
\hline Group & $\begin{array}{c}\text { No. } \\
\text { of cases }\end{array}$ & $\begin{array}{c}\text { Restenosis rate 1 month } \\
\text { after operation, cases }(\%)\end{array}$ & $\begin{array}{c}\text { Target lesion diameter } \\
\text { loss ratio, } \%\end{array}$ & $\begin{array}{c}\text { Restenosis rate 1 year } \\
\text { after operation, cases }(\%)\end{array}$ & $\begin{array}{c}\text { Target lesion diameter } \\
\text { loss rate, } \%\end{array}$ \\
\hline Control & 91 & $18(19.78)$ & $35.4 \pm 10.2$ & $23(25.27)$ & $42.3 \pm 15.4$ \\
Observation & 55 & $20(36.36)$ & $76.8 \pm 15.6$ & $28(50.91)$ & $86.5 \pm 18.2$ \\
t/ $\chi^{2}$ & & 4.897 & 9.632 & 9.911 & 13.232 \\
P-value & & 0.027 & 0.000 & 0.002 & 0.000 \\
\hline
\end{tabular}

Table IV. Multivariate logistic regression analysis.

\begin{tabular}{|c|c|c|c|c|c|}
\hline Factor & $\mathrm{B}$ & Wald & P-value & OR & $95 \% \mathrm{CI}$ \\
\hline $\mathrm{mSBP}$ & 0.063 & 4.527 & 0.037 & 1.234 & $0.635-2.532$ \\
\hline $\mathrm{mDBP}$ & 0.027 & 4.231 & 0.039 & 1.120 & $0.764-2.514$ \\
\hline Cerebral vascular stenosis rate & 0.125 & 5.203 & 0.025 & 2.635 & 2.103-3.527 \\
\hline Hyperperfusion syndrome & 0.136 & 5.639 & 0.018 & 3.205 & $2.857-3.638$ \\
\hline
\end{tabular}

B, regression coefficient; OR, odds ratio; CI, confidence interval; mSBP, mean systolic blood pressure; mDBP, mean diastolic blood pressure.

Comparison of the incidence of stent restenosis. The rate of restenosis and target lesion diameter 1 month and 1 year after operation in the observation group were significantly higher than those in the control group, and the differences were statistically significant $(\mathrm{P}<0.05$; Table III).
Comparison of the incidence of stent restenosis. We used the general information of patients including gender, age, time of disease onset, smoking, diabetes, total cholesterol, low density lipoprotein, BMI, mean systolic blood pressure (mSBP), mean diastolic blood pressure (mDBP), cerebral vascular diameter 
stenosis, stent length, high perfusion syndrome, Xe-CT parameters (blood flow of ACA, MCA, PCA, ABZ, PBZ and IBZ) as the independent variables. As a dependent variable, restenosis was included in the logistic regression model, and the method of stepwise regression was used for screening $(\alpha<0.10$ as entry criteria, $<0.05$ as exclusion criteria). We found that $\mathrm{mSBP}, \mathrm{mDBP}$, cerebral vascular stenosis rate and high perfusion syndrome were the independent risk factors for restenosis (Table IV).

\section{Discussion}

High perfusion syndrome after cerebral vascular stent implantation. CHS is a serious complication of cerebral vascular stent implantation. Cerebral vascular stenosis leads to functional decline of automatic regulation, insufficient blood volume of brain tissue, and adaptability to low perfusion pressure. After stent implantation, vascular stenosis rapidly relieves and the intracranial blood flow increases sharply, leading to a sharp increase in capillary bed perfusion pressure. These events cause the cerebrovascular system not to contract timely and effectively, which is an obstacle of the blood brain barrier mechanism and leads to brain edema and intracranial hemorrhage $(5,6)$. Occasionally the clinical symptoms are not obvious, and can easily be ignored (7).

The formation mechanism of high perfusion. High perfusion formation mechanism includes: i) Cell trauma (8): The narrow cerebral blood vessel reperfusion injury activates the ring oxidase, lipid oxidase and brain cell microsomes, which produce catalytic action to prevent acid metabolism with free radical generation. We strengthened lipid peroxidation, and changed the lipid microenvironment. This cascade of reactions continues to damage the lipid structure, resulting in cell structure and functional damage. ii) Loss of brain cell function (9): Brain cells and some of the activating enzymes have cross-linking reaction, with the formation of the two polymers, resulting in the loss of brain cells. iii) Free radical toxicity (10): Free radicals can produce toxic effects on brain cells, which can be expressed as nucleic acid base changes, chromosome aberration or DNA fault. iv) Complement activation (11): The complement terminal complex affects the brain cell function through the membrane mechanism, which triggers the release of the macrophages, and produces a variety of inflammatory factors, which can cause damage to the neurons.

Diagnosis of high perfusion syndrome. Diagnosis of high perfusion syndrome should be combined with clinical manifestations, MRA, TCD and other examinations. Among them, MRA can display intracranial and extracranial vessels, which are suitable for preoperative risk factor assessment. The abnormal manifestations include white matter edema (mainly related to occipital region), focal cerebral infarction and cerebral hemorrhage (12). TCD through cranial window can measure cerebral vascular blood flow velocity, reflect the preoperative low perfusion, brain vascular reaction, post-operative hyperperfusion and postoperative emboli formation. In clinical use, it has good diagnostic sensitivity and accuracy (13). However, due to the variation in operator experience, personal variability is very different. The human cerebral artery regulates the $\mathrm{CBF}$ through its own regulation mechanism, which is normally maintained at $50 \mathrm{ml} /(100 \mathrm{~g} \cdot \mathrm{min})$. Xe-CT can quantitatively display cerebral perfusion with convenient inspection, on the basis of routine CT examinations, fast evaluation of CBF and vascular reserve capacity, especially after stent implantation. Quantitative detection of cerebral arterial blood flow can be an effective method for diagnosis of high perfusion comprehensive syndrome of patients and improvement after operation to provide the basis for the judgment of prognosis of patients and reduce ISR $(14,15)$.

Effects of high perfusion on restenosis. After hyperperfusion syndrome, cerebral artery hemodynamics change, inducing basic fibroblast growth factor (bFGF) and platelet-derived growth factor (PDGF) expression, bFGF and PDGF may promote the division and proliferation of vascular endothelial cells, and induce migration proliferation of smooth muscle cells, ultimately lead to vascular intimal hyperplasia $(16,17)$. Puffiness has creeping growth along the stent mesh, the stent neointimal coverage area increases, so that stent restenosis occurs.

The results of the study showed that the incidence of high perfusion syndrome after stent implantation was $\sim 37.67 \%$ and that the blood flow rate of MCA, PCA, ABZ, PBZ, IBZ and ACA in patients with high perfusion syndrome significantly increased at 1 month and 1 year after operation. The rate of restenosis and target lesion diameter loss rate were significantly higher than in the observation groups compared to those in the control group. The high average systolic blood pressure and diastolic blood pressure, high brain vascular diameter stenosis rate and high perfusion comprehensive syndrome were the independent risk factors of restenosis. In conclusion, the application of Xe-CT examination has important value for early diagnosis of hyperperfusion syndrome, hyperperfusion syndrome and the occurrence of stent restenosis are closely related. Mean systolic pressure, mDBP, cerebral blood vessel diameter stenosis rate and high perfusion comprehensive syndrome are the independent risk factors of restenosis.

\section{References}

1. Gao JH, Gao XP and Pan SY: Perioperative intensive statin therapy improves outcomes in patients with ischemic stroke undergoing middle cerebral artery stent implantation. Nan Fang Yi Ke Da Xue Xue Bao 36: 356-360, 2016 (In Chinese).

2. Zhang Y, Kumar A, Tezel JB and Zhou Y: Imaging evidence for cerebral hyperperfusion syndrome after intravenous tissue plasminogen activator for acute ischemic stroke. Case Rep Neurol Med 2016: 8725494, 2016.

3. Peker A, Sorgun MH, Isikay CT, Arsava EM, Topcuoglu MA and Arat A: Perioperative and follow-up results in carotid artery stenting with contralateral carotid occlusion. Jpn J Radiol 34: 523-528, 2016.

4. Zhao Y, Jin M, Liu Q, Liu D, Chen J and Du B: A long-term follow-up results of Enterprise stent in treatment of severe symptomatic basilar artery atherosclerotic stenosis. Zhonghua Nei Ke Za Zhi 55: 372-376, 2016 (In Chinese).

5. Guirgis M and Sieunarine K: Cerebral hyperperfusion syndrome: A rare postoperative complication of carotid endarterectomy. Am J Emerg Med: Mar 5, 2016 (Epub ahead of print).

6. Pinho J, Amorim JM, Alves JN, Araújo JM and Ferreira C: Cerebral hyperperfusion syndrome after internal carotid artery dissection with persistent occlusion. Neurol Sci 37: 1175-1177, 2016.

7. Uchida K, Yoshimura S, Shirakawa M, Shindo S, Egashira Y, Iwama T and Yamada K: Experience of Staged Angioplasty to Avoid Hyperperfusion Syndrome for Carotid Artery Stenosis. Neurol Med Chir (Tokyo) 55: 824-829, 2015. 
8. Chadha DS, Singh N, Tewari AK, Kumar RS, Yadav KK, Naveen AJ, Bhartiya M, Gupta VK, Wagh A and Ghosh AK: Hyperperfusion Syndrome after Carotid Artery Stenting. Med J Armed Forces India 71 (Suppl 1): S156-S159, 2015.

9. Aralasmak A, Atay M, Toprak H, Ozdemir T, Asil T, Goktekin O, Mehdi E, Ozdemir H and Alkan A: Hyperperfusion in carotid stenting patients. J Comput Assist Tomogr 39: 313-316, 2015.

10. Oh SI, Lee SJ, Lee YJ and Kim HJ: Delayed cerebral hyperperfusion syndrome three weeks after carotid artery stenting presenting as status epilepticus. J Korean Neurosurg Soc 56 441-443, 2014.

11. Xu S, Wu P, Shi H, Ji Z and Dai J: Hyperperfusion Syndrome After Stenting for Intracranial Artery Stenosis. Cell Biochem Biophys 71: 1537-1542, 2015

12. Sato K, Yamada M, Kuroda H, Yamamoto D, Asano Y, Inoue $Y$, Fujii $\mathrm{K}$ and Kumabe T: Time-of-flight MR angiography for detection of cerebral hyperperfusion syndrome after superficial temporal artery-middle cerebral artery anastomosis in moyamoya disease. Am J Neuroradiol 37: 1244-1248, 2016.

13. Orion D, Yavne Y, Peretz S and Givaty G: Diagnosing hyperperfusion syndrome: CT perfusion or transcranial doppler? Isr Med Assoc J 17: 656-658, 2015.
14. Yoshie T, Ueda T, Takada T, Nogoshi S, Fukano T and Hasegawa Y: Prediction of cerebral hyperperfusion syndrome after carotid artery stenting by CT perfusion imaging with acetazolamide challenge. Neuroradiology 58: 253-259, 2016.

15. Schoknecht K, Gabi S, Ifergane G, Friedman A and Shelef I: Detection of cerebral hyperperfusion syndrome after carotid endarterectomy with CT perfusion. J Neuroimaging 24: 295-297, 2014.

16. Osadnik T, Strzelczyk JK, Reguła R, Bujak K, Fronczek M, Gonera M, Gawlita M, Wasilewski J, Lekston A, Kurek A, et al: The relationships between polymorphisms in genes encoding the growth factors TGF- $\beta 1$, PDGFB, EGF, bFGF and VEGF-A and the restenosis process in patients with stable coronary artery disease treated with bare metal stent. PLoS One 11: e0150500, 2016.

17. Guildford AL, Stewart HJ, Morris C and Santin M: Substrateinduced phenotypic switches of human smooth muscle cells: An in vitro study of in-stent restenosis activation pathways. J R Soc Interface 8: 641-649, 2011. 